\title{
II. Innenpolitik / Aussenpolitik
}

\section{(2) OpenEdition \\ 12 Journals}

Electronic version

URL: http://journals.openedition.org/sjep/1023

DOI: $10.4000 /$ sjep.1023

ISSN: 1663-9677

\section{Publisher}

Institut de hautes études internationales et du développement

\section{Printed version}

Date of publication: 1 janvier 1985

Number of pages: 65-69

ISSN: 1660-5926

\section{Electronic reference}

«II. Innenpolitik / Aussenpolitik», Schweizerisches Jahrbuch für Entwicklungspolitik [Online], 5 | 1985,

Online erschienen am: 16 Februar 2013, abgerufen am 08 September 2020. URL : http://

journals.openedition.org/sjep/1023 ; DOI : https://doi.org/10.4000/sjep.1023 


\section{INNENPOLITIK / AUSSENPOLITIK}

\section{ZWEITE REVISION DES ASYLGESETZES}

Die Schweiz hat seit 1979 ein einheitliches Asylgesetz. Am 1. Juni 1984 trat eine revidierte Fassung in Kraft. Das revidierte Gesetz sollte durch eine Vereinfachung der Behandlung die Erledigung der zahlreichen Asylgesuche beschleunigen. Seither verlangten verschiedene parlamentarische Vorstösse zur schweizerischen Asylpolitik bereits wieder eine neue Teilrevision des Asylgesetzes, um die Attraktivität der Schweiz als Asylland weiter zu vermindern. Im Juli 1985 wurde der Entwurf zur zweiten Revision des Asylgesetzes in die Vernehmlassung geschickt.

Der Artikel von M.-C. Caloz-Tschopp im zweiten Teil dieses Buches befasst sich mit dem Wandel in der schweizerischen Asylpolitik angesichts der neuen Flüchtlingsströme aus Ländern der Dritten Welt.

Im Zentrum der öffentlichen Diskussion und der Medienberichterstattung stand die Zunahme der Asylgesuche von Tamilen aus Sri Lanka und die damit im Zusammenhang stehenden Probleme einer Zunahme der Fremdenfeindlichkeit. Ein durch Indiskretion bekannt gewordener, als "vertraulich" qualifizierter Bericht der Bundesanwaltschaft (zuhanden der kantonalen Polizeikommandanten) zur Asylpolitik löste heftige Reaktionen und Empörung aus. Parteien und Hilfswerke verurteilten die "pauschale Verunglimpfung" (1) von Asylsuchenden durch die Verfasser des Berichts. Der Bericht enthält eine Aufzählung von Missbrauchspraktiken im Asylwesen.

Ende April 1985 waren 13661 Asylgesuche erstinstanzlich (Bundesamt für Polizeiwesen) noch nicht entschieden. Dazu waren beim EJPD und beim Bundesrat 8333 Beschwerden hängig. Insgesamt lebten 30962 anerkannte Flüchtlinge in der Schweiz. Von den 1984 eingereichten 7435 Asylgesuchen (Vorjahr 7886) stammten 2639 Bewerber aus der Türkei und 1236 aus Sri Lanka (2).

Ein Bericht des EJPD über die Situation der Tamilen in Sri Lanka löste eine heftige Kontroverse aus. Nach Aussage des Berichts bestehe für Tamilen in Sri Lanka keine generelle Gefahr, so dass Tamilen künftig in ihr Land zurückgeschickt werden könnten. Der Bundesrat entschied daraufhin im Oktober 1984, Tamilen ohne anerkannte Flüchtlingseigenschaft könnten grundsätzlich nach Sri Lanka zurückgeschickt werden. Die Heimschaffung von Tamilen wurde im Dezember gleichen Jahres angesichts der verschärften Situation in Sri Lanka gestoppt. 


\section{Parlamentsdebatte}

Der Ständerat überwies an seiner Märzsession eine vom Nationalrat bereits im Juni 1984 gutgeheissene Motion (Lüchinger, FDP) zur Revision des Asylgesetzes. Die Motion hat folgenden Inhalt: Der Bundesrat wird eingeladen, der Bundesversammlung eine neue Vorlage zur Revision des Asylgesetzes vorzulegen, welche die verfahrensmässige Behandlung von Asylgesuchen - insbesondere bei grossem Andrang von Asylbewerbern - weiter vereinfachen und dem Bundesrat eine grössere Beweglichkeit zur Meisterung ausserordentlicher Situationen einräumen soll (3). Damit wurde das EJDP mit der Ausarbeitung eines Revisionsentwurfs für die Vernehmlassung beauftragt (Vernehmlassungsfrist bis 30. September 1985).

Im Rahmen einer vertieften asylpolitischen Aussprache genehmigte der Bundesrat im Juni vor dem Beginn der Parlaments-Sommersession die Grundzüge der zweiten Asylgesetzrevision: Massnahmen zur weiteren Vereinfachung und Beschleunigung der Asylverfahren, verbunden mit einer konsequenten Wegweisungspraxis und einer verbesserten Zusammenarbeit mit den Kantonen.

Das EJPD wurde beauftragt, mit den Kantonen über eine Globallösung für alte Asylgesuche zu verhandeln. Einige Kantone hatten zu restriktiven Asylpraktiken gegriffen. So verbot der Kanton Bern beispielsweise die Arbeitsbewilligung für Asylanten. Die Praxis im Kanton Freiburg, keine Asylgesuche mehr entgegenzunehmen, widersprach dem Asylgesetz. Im Kanton Graubünden wurden von den Asylbewerbern ohne Rechtsgrundlage Kautionen erhoben, um die Abschiebung zu finanzieren.

Ein Positionspapier der SVP (Schweizerischen Volkspartei) zur Asylpolitik setzte sich für eine "Kantonalisierung" des Asylverfahrens ein. Künftig sollen die Kantone in erster Instanz entscheiden. Zudem soll bereits weitgehend assimilierten Asylbewerbern kollektiv Asyl gewährt werden. Die bürgerlichen Parteien FDP und CVP befürworteten eine Globallösung; die SP steht dieser Idee aus rechtsstaatlichen Gründen ablehnend gegenüber.

\section{Revisionsentwurf}

Der Revisionsentwurf, wie er im Juli 1985 in die Vernehmlassung geschickt wurde, sieht folgende Revisionspunkte vor:

Vereinfachung des Verfahrens: Die Asylbewerber sollen nur noch in einer Befragung (bisher zwei) zu den Gründen ihrer Flucht befragt werden. Nach der zweiten Revision soll das Bundesamt für Polizeiwesen auf eine eigene Befragung verzichten und auf Grund der Akten entscheiden können. Dagegen hätten die kantonalen Behörden die Asylgesuchsteller eingehend und detailliert zu den Asylgründen zu befragen.

Ausschaffungshaft: Für Asylbewerber mit ablehnendem Entscheid, die sich einer Wegweisung widersetzen, soll die Ausschaffungshaft eingeführt werden (maximal 30 Tage). Dieser Punkt würde eine Legalisierung der heute üblichen Praxis bedeuten. 
Rückkehrhilfen: Der Bund gewährt Asylgesuchstellern Rückkehrhilfen und Wiedereingliederungsbeiträge. Finanzielle Unterstützung soll die Rückkehr in die Heimat oder in ein Land des Kulturkreises des Asylanten ermöglichen.

Notrecht: Das Bundesrat ersucht im Revisionsentwurf um die Kompetenz von Notrecht, um für den Fall eines plötzlichen und massiven Ansteigens der Asylbewerberzahlen restriktiv reagieren zu können.

Aufnahmepflicht der Kantone: Der Entwurf sieht eine subsidiäre Bundeskompetenz zur Verteilung der Asylbewerber auf die Kantone vor, falls Verhandlungen zwischen den Kantonen gescheitert sind.

Globale Erledigung von Gesuchen: Asylbewerber, die vor 1982 in die Schweiz eingereist sind und auf ihr Asylgesuch noch keinen Entscheid erhalten haben, sollen eine normale fremdenpolizeiliche Aufenthaltsbewilligung erhalten. Diese Regelung ist vor a!lem für Familien vorgesehen.

\section{Vorschläge der Hilfswerke}

Die der Schweizerischen Zentralstelle für Flüchtlingshilfe angeschlossenen Hilfswerke riefen im Mai 1984 die Parlamentarier in einem Brief auf, von einer weiteren Revision des Asylgesetzes abzusehen. Sie schlugen Massnahmen zur Beschleunigung des Verfahrens bei der Erledigung von Asylgesuchen vor, die inzwischen zum Teil in der zweiten Revisionsvorlage enthalten sind (Zusammenlegung der kantonalen und der eidgenössischen Befragung zu den Fluchtgründen, Rückkehrberatung und -hilfe).

Die Hilfswerke wehrten sich beim Bundesrat gegen die Heimschaffung von Tamilen nach Sri Lanka. In einem eigenen Bericht über die Lage in Sri Lanka kamen die Hilfswerke entgegen der Meinung des Bundesrates zum Schluss, dass eine Rückführung von Tamilen nicht verantwortbar sei. Ausgehend von den besonderen Problemen der srilankischen Tamilen machten die Hilfswerke den Vorschlag des "Asyls auf Zeit", befristet auf vorläufig 1 Jahr, das für sinnvolle Rückkehrvorbereitungen genutzt werden soll.

Die Schweizerische Liga für Menschenrechte lud im Februar 1985 zahlreiche Vertreter aus dem In- und Ausland zu einem Erfahrungsaustausch über die verschlechterte Lage der Asylanten in Europa nach Lausanne. Das Symposium beschloss, sich gemeinsam für eine humanere Asylrechtspolitik einzusetzen (4).

\section{Anmerkungen}

1) Kritik des Schweizerischen Arbeiterhilfswerks, SAH, zit. in NZZ vom 6.6.1985.

2) Zahlen vom EJPD, Ausländer- und Asylstatistik Ende 1984 bzw. Ende April 1985.

3) Motion Lüchinger (FDP), zit. in NZZ 12.3.1985.

4) Die Akten wurden publiziert in: "La Forteresse Européenne et les Réfugiés", Lausanne 1985. 


\section{Quellen}

EJPD, Asylgesetzrevision: Eröffnung des Vernehmlassungsverfahrens, Pressemitteilung vom 8.7.1985.

EJPD, Vorentwurf und Bericht zur Revision des Asylgesetzes, Bern, Juli 1985.

Schweizerische Zentralstelle für Flüchtlingshilfe, Pressemitteilung vom 28.5.1984.

Caritas Schweiz/Fastenopfer/Brot für Brüder/Helvetas/Swissaid/HEKS, Pressemittei-

lung vom 28.9.1984.

Die Wochenzeitung, 14. Juni 1985.

NZZ 12.3., 4.6. und 6.6.1985.

TA 22.5., 4.6. und 6.6.1985.

\section{BEITRITT DER SCHWEIZ ZUR UNO}

1984 haben beide Räte die Beratungen über einen Beitritt der Schweiz zur UNO abgeschlossen. Im Frühjahr beschloss der Nationalrat und in der Wintersession auch der Ständerat den Beitritt der Schweiz zur UNO. Die Volksabstimmung wird im Frühjahr 1986 stattfinden.

Am 15. März 1984 entschied der Nationalrat mit $112: 78$ Stimmen, der UNO beizutreten. Die schweizerische Neutralität soll gemäss diesem Beschluss mit verschiedenen Erklärungen des Bundesrates vor einer Aufnahme der Schweiz in die UNO abgesichert werden (1).

\section{Debatte im Ständerat}

Hauptargument gegen einen UNO-Beitritt blieb auch in der Debatte im Ständerat die Neutralitätsfrage. Die Gegner eines UNO-Beitritts sahen die Neutralität gefährdet, wenn die Schweiz in der UNO mitarbeiten werde. Die UNO als "hochpolitische Organisation" (2) und die schweizerische Neutralität liessen sich ihrer Ansicht nach nicht in Einklang bringen. Als Vertreter der Befürworter bezeichnete Kommissionspräsident Ständerat Muheim seinerseits die Aussenpolitik als eines der staatlichen Mittel zur Sicherung der territorialen Existenz, der politischen Unabhängigkeit und der kulturellen Identität. Die Neutralität diene zur Verteidigung dieser Staatszwecke; sie sei aber kein in der Verfassung genannter Bundeszweck, sondern ein sich den veränderten Umweltbedingungen anzupassendes Instrument. Für die Befürworter eines UNO-Beitritts bieten die ebenfalls neutralen Staaten Schweden, Finnland und Oesterreich das Beispiel, dass auch neutrale Staaten aktiv in der UNO mitarbeiten können.

Als weiteres Argument für einen UNO-Beitritt wurde in der Debatte die Gestaltung des internationalen Wirtschaftsrechts und des Völkerrechts genannt. Als UNO-Mitglied könne die Schweiz aktiv an der Weitergestaltung internationaler Rechtsnormen arbeiten, welche durch einen UNO-Beitritt auch für die Schweiz verbindlich würden. 
Als UNO-Mitglied könne sich die Schweiz zu wichtigen Fragen in der UNGeneralversammlung äussern und direkt auf die Aktivitäten der UNO Einfluss nehmen, was die Schweiz als Land mit Beobachterstatus nicht könne. Nach zweitägiger Debatte befürwortete der Ständerat den Beitritt der Schweiz zur UNO mit 24 : 16 Stimmen.

Die Volksabstimmung findet am 16. März 1986 statt.

\section{Anmerkungen}

1) Die UNO, 1945 gegründet, zählt heute 159 Mitgliedstaaten. Die Schweiz ist bei den Vereinten Nationen durch einen ständigen Botschafter vertreten (Botschafterin Francesca Pometta). Die Schweiz ist Mitglied in vielen UN-Sonderorganisationen.

2) Ständerat Affolter (FDP), Sprecher der Minderheit gegen einen UNO-Beitritt, in der Debatte im Ständerat, zit. in NZZ 13.12.1984.

\section{Quellen}

Botschaft des Bundesrates zum Beitritt der Schweiz zur UNO, 21. Dezember 1981. Bericht des Bundesrates über seine Geschäftsführung im Jahre 1984, Bern, Februar 1985.

NZZ, 13. und 14.12.1984.

TA 24.10.1984, 13. und 14.12.1984. 